\title{
Phase Equilibrium, Structure, and Rheological Properties of the Carboxymethyl Cellulose-Water System ${ }^{1}$
}

\author{
S. A. Vshivkov* and A. A. Byzov \\ Ural Federal University, pr. Lenina 51, Yekaterinburg, 620000 Russia \\ *e-mail: sergey.vshivkov@usu.ru \\ Received June 27, 2012; \\ Revised Manuscript Received August 2, 2012
}

\begin{abstract}
The phase transitions, structure, and rheological properties of the carboxymethyl cellulose-water system were studied via the turbidity-point method, viscometry, polarization microscopy, and the turbidityspectrum method as well as with a polarization photoelectric unit. The regions of existence of the isotropic and anisotropic phases, the gel point, and the concentration dependence of the supramolecular-particle size were determined. Magnetic-field application results in a gain in the viscosities of carboxymethyl cellulose solutions.
\end{abstract}

DOI: $10.1134 / \mathrm{S} 0965545 \mathrm{X} 13020107$

In recent years, the phase transitions, structure, and rheological properties of solutions of rigid-chain polymers (cellulose ethers) have been studied at the Chair of Macromolecular Compounds, Ural Federal University [1-7]. The molecules of cellulose and its derivatives are characteristic of a rigid helical conformation and capable of ordering via the formation of cholesteric liquid crystals in concentrated solutions [8]. The additional orientation of these macromolecules induced by a magnetic or mechanical field is responsible for broadening of the temperature-concentration region of LC-phase existence and additional organization of macromolecules $[1,4,6,7]$. The first studies of the viscosity of rigid-chain polymer solutions are described in [9-13]. Note that the data on the effect of a magnetic field on the viscosity of a polymer solution are few.

The aim of this study was to investigate the phase equilibrium and structure of carboxymethyl cellulose aqueous solutions as well as their rheological properties in the presence and the absence of a magnetic field.

\section{EXPERIMENTAL}

The experiments were performed with carboxymethyl cellulose $(\mathrm{CMC}) 7 \mathrm{M}$ (Aqualon/Hercules, $M_{\eta}=$ $1.2 \times 10^{5}$, a substitution degree of 0.7 ). The purity of bidistilled water used as a solvent was confirmed by the refractive-index value [14]. Solutions were prepared for 30-40 days at $293 \mathrm{~K}$.

\footnotetext{
${ }^{1}$ This work was supported by the Russian Foundation for Basic Research (project no. 12-08-0038-a).
}

The type of phase transition in a solution was determined on a polarization photoelectric unit $[1,15]$. An ampoule with a polymer solution was placed in a gap between crossed polaroids and the solution temperature was lowered with a thermostatically controlled jacket. The appearance of turbidity with cooling of the system is accompanied by an increase in the intensity of light transmission. This phenomenon suggested the anisotropic character of the forming phase, i.e., an LC-phase transition occurs. Melting temperatures of gels were determined as follows: Ampoules with gels were turned so that gels were located at the top, the system was heated slowly $(\sim 1 \mathrm{~K} / \mathrm{h})$, and the temperature of flow onset was fixed [1]. The phase states of solutions and gels were studied with an Olympus BX-51 polarization microscope.

The radii of supramolecular particles, $r_{\mathrm{w}}$, in moderately concentrated and concentrated solutions were determined through the method of the turbidity spectrum, proposed by W. Heller et al. [16, 17] and developed by V.I. Klenin et al. [18]. The method is based on the Angström equation $A \sim \lambda^{-n}$, where $A$ is the optical density of the solution; $\lambda$ is the wavelength of transmitting light; and exponent $n$ is dependent on the relative refractive index of the solution, $m_{\text {rel }}$, and the $\alpha$ coefficient, related to light-scattering-particle size. For each solution, the $\ln A-\ln \lambda$ dependences were plotted, while the $n$ value was found from the line slope.

The optical densities of solutions were measured on a KFK-3 spectrophotometer. The relative refractive indexes were calculated through the equation $m_{\text {rel }}=$ $n_{d \mathrm{pol}} / n_{d \mathrm{~s}}$, where $n_{d \mathrm{pol}}$ and $n_{d \mathrm{~s}}$ are the refractive indexes of the polymer and the solvent, respectively. Literature data on $n_{d}$ for CMC are absent. Thus, this value was 
calculated through the Vogel method [19]: $\mathrm{MR}=n_{d \mathrm{pol}} m$, where $m$ is molecular mass of a unit, $n_{d \mathrm{pol}}$ is the refractive index of the polymer, and MR is the molecular refraction. The calculated mass of the CMC unit with a substitution degree of 0.7 is $m_{\text {un }}=202$. The group contributions to molecular refraction for $\lambda=589 \mathrm{~nm}$ were taken from [19]: $\mathrm{R}_{\mathrm{CH}_{2}}=20.64, \mathrm{R}_{\mathrm{CH}}=23.49$, $\mathrm{R}_{\mathrm{O} \text { acetal }}=22.99, \mathrm{R}_{\mathrm{OH} \mathrm{sec}}=23.95$, and $\mathrm{R}_{\mathrm{O} \text { ether }}=23.18$. The molecular refraction of CMC calculated from these data is $\mathrm{MR}=253.47$, and refractive index calculated from these data is $n_{d}=\mathrm{MR} / m=1.253$. The obtained value of $n_{d \mathrm{CMC}}$ is in satisfactory accordance with the refractive indexes for other cellulose ethers [20]. With the use of tables from [18], parameters $\alpha$ for the determined values of $m_{\text {rel }}$ and $n$ were found. Parameter $\alpha$ is related to the weight-average radius of light-scattering particles through the expression $\alpha=$ $2 \pi \mathrm{r}_{\mathrm{w}} / \bar{\lambda}$. In this relationship, $\bar{\lambda}$ is the wavelength of light transmitting through the solution, which is equal to $\bar{\lambda}=\lambda_{\mathrm{av}} / n_{d \mathrm{~s}}$, where $\lambda_{\mathrm{av}}$ is the wavelength of light in vacuum corresponding to the middle of the linear portion of the $\ln A-\ln \lambda$ plot. This method allows determination of the light-scattering-particle radii in the range $30-3000 \mathrm{~nm}$.

For calculation of the macromolecule size, a Kuhn segment value of $\mathrm{A}=20.2 \AA$ [8], a length of the cellobiose residue of $1.03, \mathrm{~nm}$, and a length of the cellulose ether macromolecule unit of $0.5 \mathrm{~nm}$ were used. The contour length of the macromolecule was calculated via the equation $L=0.5 n$, where $n$ is the degree of polymerization. The number of Kuhn segments in the macromolecule was found via the equation $N=L / A$, where $A$ is the Kuhn segment length. The meansquare distance between the macromolecule-chain ends was calculated via the equation $\left(h^{2}\right)^{(1 / 2)}=A N^{(1 / 2)}$.

Solution viscosity was measured on a modified Rheotest RN 4.1 rheometer with a cylindrical working unit made from a low-magnetic material, such as brass. The magnetic-field effect on the rheological properties of solutions was studied with the use of a magnet inducing a permanent magnetic field with an intensity of $3.7 \mathrm{kOe}$ and lines of force perpendicular to the rotational axis of a rotor. The working unit with a solution was placed into the magnetic field at $298 \mathrm{~K}$ and kept for $20 \mathrm{~min}$, and the viscosity in the presence of the magnetic field was measured at different shear rates.

The metal rotor rotating in the magnetic field can be considered a generator closed on itself [21]. During generator duty, so-called braking electromagnetic moment $M_{\mathrm{e}}$ is induced. As a result, during measurement of the shear stress of deformed solutions, the fixed stress value exceeds the real value by a value related to electromagnetic moment [21]:

$$
M_{\mathrm{e}}=\frac{p N}{2 \pi 9.81 a} \Phi I_{\mathrm{an}}=K \Phi I_{\mathrm{an}},
$$

where $p$ is the number of pole pairs, $N$ is the number of coil conductors, $2 a$ is the number of parallel branches, $\Phi$ is the magnetic flux, and $I_{\text {an }}$ is the anchor current. For a given unit, $p, N$, and $2 a$ are constants; thus, $K=$ $\frac{p N}{2 \pi 9.81 a}$ is likewise constant. The magnetic flux was determined as [21]

$$
\Phi=B S \cos \alpha
$$

where $B$ is the magnetic induction, $S$ is the area of the contour crossed by magnetic-induction vectors, and $\alpha$ is the angle between the magnetic-induction vector and the normal to the contour surface. Because the anchor is closed on itself,

$$
I_{\text {an }}=\frac{E}{R}=\frac{p N}{60 a R} n \Phi,
$$

where $E$ is the emf of the generator, $R$ is the electric resistance of the anchor, and $n$ is rotation rate of the rotor anchor. After obvious transformations, the following calculation formula was obtained:

$$
\begin{gathered}
M_{\mathrm{e}}=K^{\prime} \mu^{2} H^{2} n, \\
\text { at } K^{\prime}=\frac{p^{2} N^{2}}{120 \pi 9.81 a^{2} R} \mu_{0}^{2} S^{2} \cos ^{2} \alpha
\end{gathered}
$$

The magnetic-field intensity is constant. The values of magnetic permeability $\mu$ for some low-magnetic substances at $293 \mathrm{~K}$ are given below [22].

$$
\begin{array}{cccc}
\text { Substance } & \text { Air } & \text { Water } & \text { Nitrogen } \\
(\mu-1) \times 10^{6} & 0.3 & -9.1 & -0.0074
\end{array}
$$

The magnetic permeabilities of substances differ from 1 by five to eight decimal digits. Therefore, a change in the medium should not affect the electromagnetic moment, which depends only on the rotation rate of the rotor.

For consideration of the electromagnetic moment, a correction dependence was plotted for shear stress versus shear rate in a working unit with the cylinder surfaces separated by air (Fig. 1). Analogous measurements were conducted for water and DMF. All the data on the electromagnetic moment agreed. The real shear stress for a solution was obtained as the difference between the measured and correction values for the same shear rate.

\section{RESULTS AND DISCUSSION}

Figure 2 shows the experimental results on phase transitions in the CMC-water system. It is evident that, at $298 \mathrm{~K}, \mathrm{CMC}$ solutions are isotropic in the composition range $0<\omega_{2}<0.08$ and anisotropic in the composition range $\omega_{2}>0.08$, where $\omega_{2}$ is the CMC weight fraction in the system. Note that, at room temperature, the concentrations of isotropic phase $\rightarrow$ anisotropic phase and solution $\rightarrow$ gel transitions are 


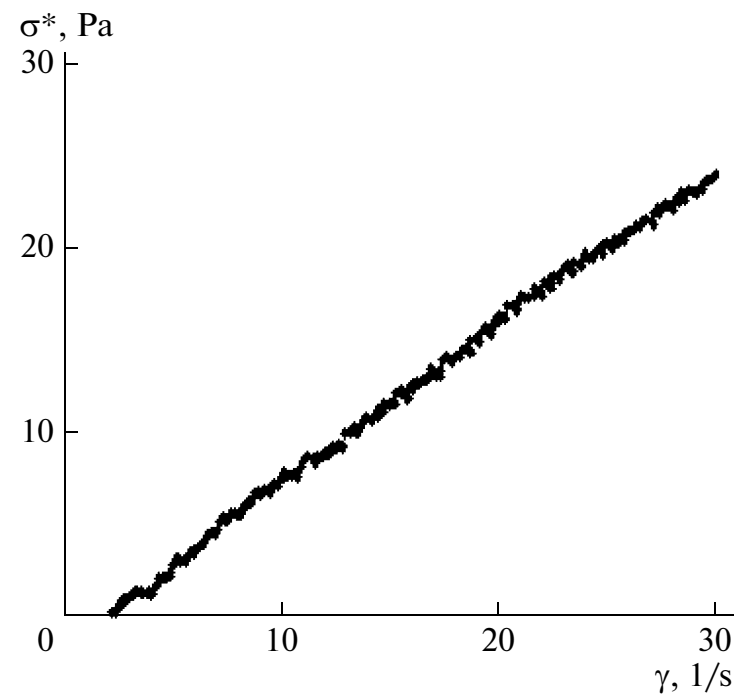

Fig. 1. Shear stress vs. rate for the working unit with air (correction plot): $H=3.7 \mathrm{kOe}$.

practically coincident. The anisotropic character of $\mathrm{CMC}$ gels is confirmed by the iridescent color of their microscopic images recorded with crossed polaroids. During cooling, turbidity in the CMC-water system was not observed, a result that is related to a small difference in the refractive indexes of the components, $\Delta n=0.08$.

Figure 3 demonstrates the concentration dependence of the optical density of the CMC-water system. The optical density grows with the concentration. This result is indicative of the system structuring,

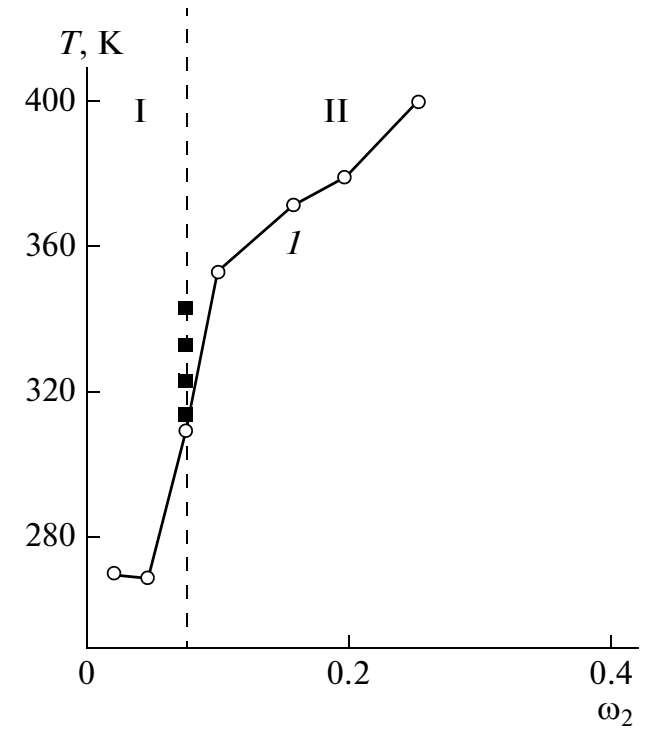

Fig. 2. Phase state and gel point for the $\mathrm{CMC}-$ water system: I and II are the isotropic region and the anisotropic region, respectively; (1) gelation curve; $\omega_{2}$ is the weight fraction of CMC in the system. Dark points correspond to the concentration of the isotropic $\rightarrow$ anisotropic solution transition at a given temperature. which must be manifested in an increase in the radii of light-scattering particles, $\mathbf{r}_{\mathrm{w}}$. It follows from the comparison with the phase diagram that the sharpest increase in optical density is observed during LCphase formation.

Figure 4 shows the $\mathbf{r}_{\mathrm{w}}$ values determined via the method of the turbidity spectrum. The following values of the light-scattering-particle diameter $D=2 \mathbf{r}_{\mathrm{w}}$ in dilute solutions $\left(\omega_{2}=0.01\right)$; calculated contour length $L$; the number of Kuhn segments in a macromolecule, $N$; and the mean-square distance between chain ends, $\left(h^{2}\right)^{1 / 2}$, were obtained: $D=100, L=305 \mathrm{~nm}, N=15$, and $\left(h^{2}\right)^{1 / 2}=80 \mathrm{~nm}$. The comparison of the above data and the data from Fig. 4 suggests that individual macromolecules exist in dilute solutions $\left(\omega_{2}<0.01\right)$, while macromolecular associates exist in moderately concentrated and concentrated solutions. The associate size increases with the polymer concentration. The sharpest increase in the light-scattering-particle size is observed in the anisotropic region.

Figure 5 demonstrates typical curves of the viscosity of a $\mathrm{CMC}$ aqueous solution versus shear rate. CMC solutions are non-Newtonian liquids. This fact correlates with the literature data for other LC systems [4$9,21,22]$ and is indicative of the disruption of the pristine structure of polymer solutions and macromolecular orientation in the flow direction during deformation.

From Fig. 5, it follows that the application of the magnetic field results in an increase in the viscosities of CMC aqueous solutions. This result may be related to additional organization of macromolecules owing

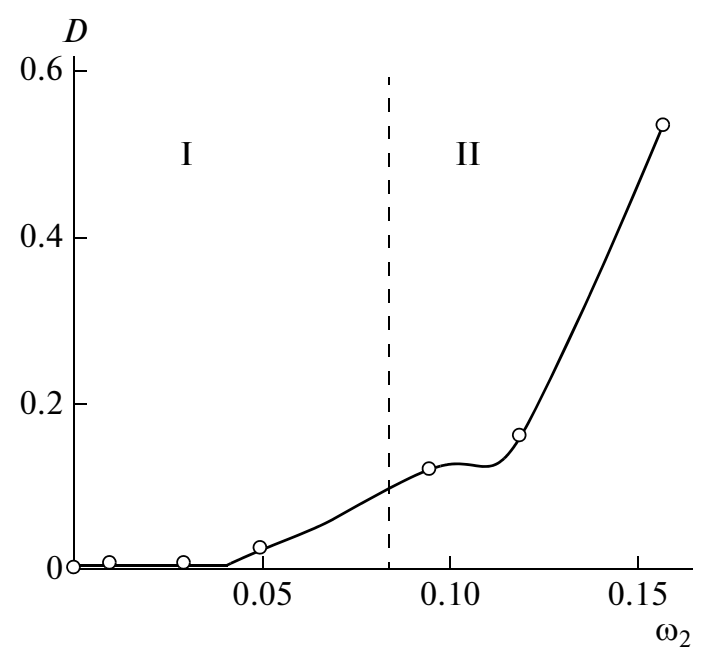

Fig. 3. Concentration dependence of optical density of the $\mathrm{CMC}-$ water system: I and II are the isotropic region and the anisotropic region, respectively: $\lambda=490 \mathrm{~nm} ; T=$ $298 \mathrm{~K}$. 


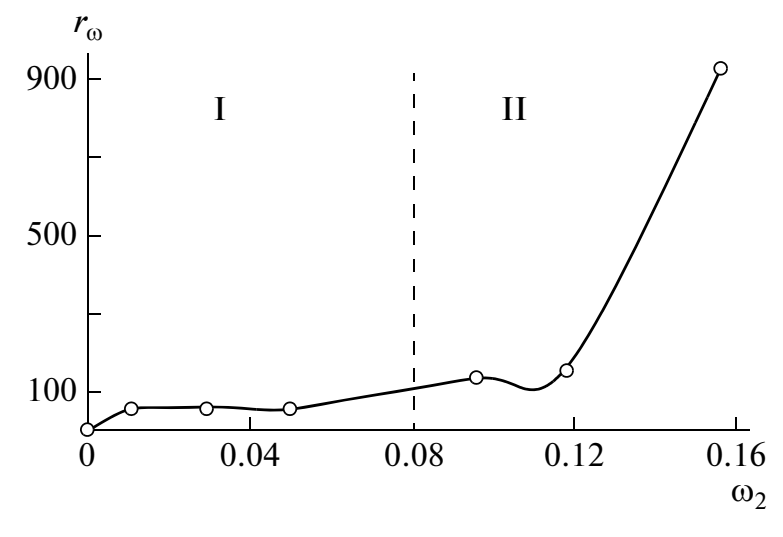

Fig. 4. Concentration dependence of the light-scatteringparticle radius of the CMC-water system: I and II are the isotropic region and the anisotropic region, respectively.

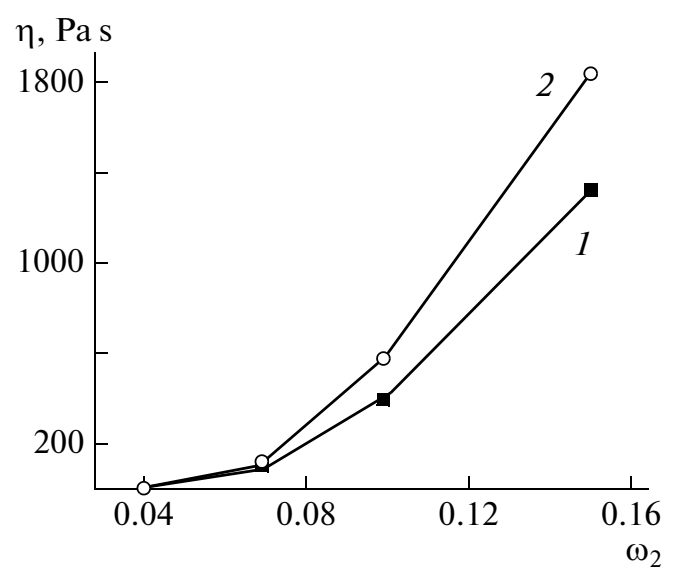

Fig. 6. Concentration dependence of the viscosity of the CMC-water system: (1) in the absence and (2) in the presence of the magnetic field; $\gamma=2.5 \mathrm{~s}^{-1}$.

to their orientation in the magnetic field. In the magnetic field, macromolecules are oriented with long chains in parallel to the lines of force [23]. Note that such an orientation is related not to the presence of permanent magnetic domains but to the molecular diamagnetic anisotropy of macromolecules. This circumstance leads to the formation of supramolecular particles, especially in the vicinity of the LC-phase transition $[4,6,7]$.

The results were used to plot the concentration dependence of viscosity. For this purpose, viscosity values at low shear rates were taken because it was shown previously [9-11] that the concentration dependence of viscosity at low shear rates is typical of anisotropic solutions (Fig. 6). The processes that occur during solution flow in a magnetic field can be described with the scheme presented in Fig. 7. In quadrants I and III, the orientation of macromolecules coincides with the flow direction and viscosity

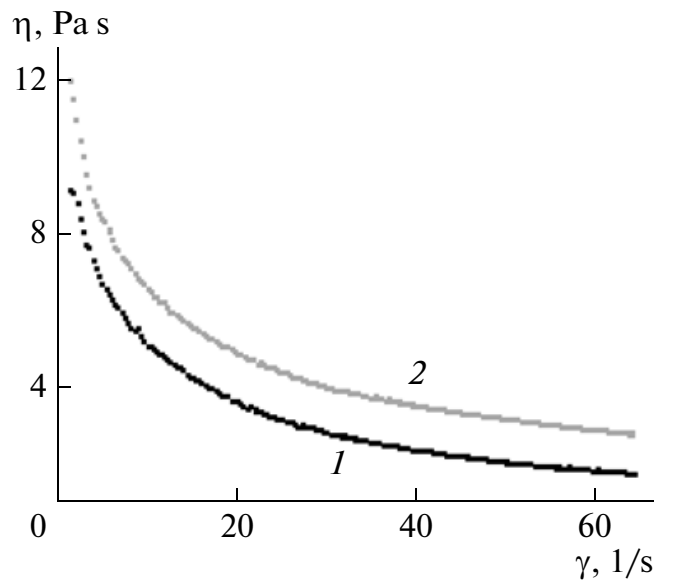

Fig. 5. Viscosity of the CMC aqueous solution vs. shear rate: (1) in the absence and (2) in the presence of the magnetic field; $\omega_{2}=0.04$.

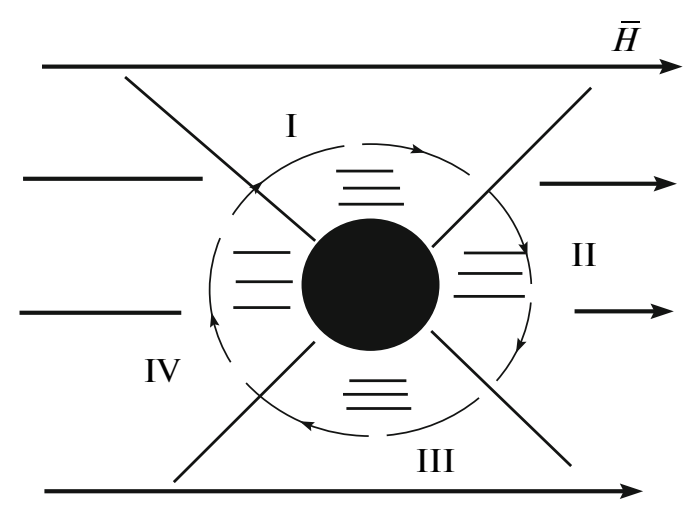

Fig. 7. Scheme of solution flow in the magnetic field with lines of force perpendicular to the rotation axis of the rotor (top view).

can decrease. In quadrants II and IV, the macromolecules are oriented perpendicularly to the flow direction and viscosity must grow. In general, as was shown experimentally, the viscosity of a solution in the magnetic field increases particularly owing to the possible self-organization of macromolecules.

Figure 8 shows the concentration dependence of $\eta / \eta_{0}$ for the CMC-water system, where $\eta$ and $\eta_{0}$ are the solution viscosities in the presence and the absence of the magnetic field, respectively. It is evident that, in the studied composition range, $\eta / \eta_{0}>1$ and the concentration dependence of this value is described by a curve with a maximum. In dilute solutions, macromolecules are few and, hence, the field effect is insignificant. The number of macromolecules capable of orientation in the magnetic field increases with the polymer concentration, and the field effect on the system properties becomes stronger. However, at high concentrations, the density of the fluctuation network 


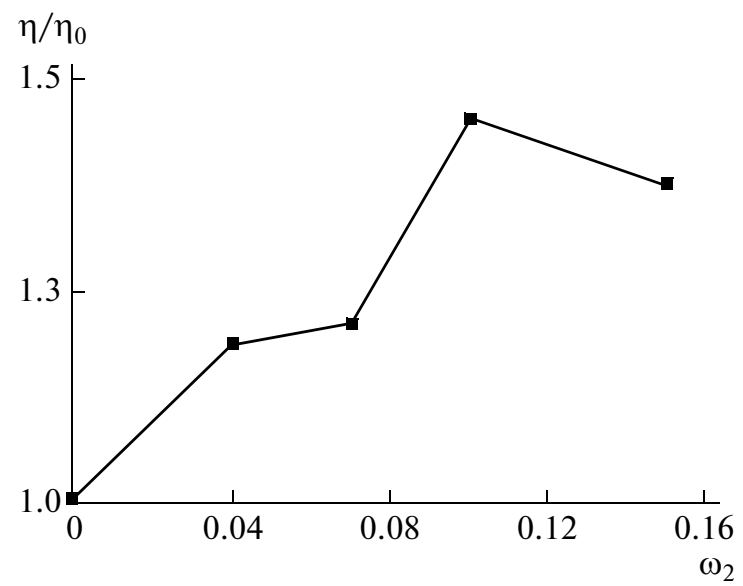

Fig. 8. Concentration dependence of $\eta / \eta_{0}$ for the CMCwater system, where $\eta$ and $\eta_{0}$ are the values of system viscosity in the presence and in the absence of the magnetic field, respectively; $\gamma=2.5 \mathrm{~s}^{-1}$.

of entanglements increases, thereby hindering the orientation processes. A similar phenomenon was observed [1,24] for concentration dependences of the supramolecular particle size and the value $\Delta T=T_{\mathrm{p} 1}-$ $T_{\mathrm{p} 2}$ in the magnetic field, where $T_{\mathrm{p} 1}$ and $T_{\mathrm{p} 2}$ are the temperatures of LC-phase transitions in the presence and the absence of the magnetic field, respectively.

Thus, in this study, the macromolecule size for the CMC-water system was calculated, the regions of isotropic- and anisotropic-phase existence were determined, and the concentration dependence of the supramolecular particle size was found. With an increase in the $\mathrm{CMC}$ concentration, macromolecular associates form, a phenomenon that manifests itself in an increase in the light-scattering-particle size. The rheological properties of the system in the presence and the absence of a magnetic field were studied, and it was found that, in a magnetic field, the viscosities of CMC solutions grow.

\section{REFERENCES}

1. S. A. Vshivkov, Phase Transitions of Polymer Systems Under External Fields (AMB, Yekaterinburg, 2011) [in Russian].

2. S. A. Vshivkov and E. V. Rusinova, Polymer Science, Ser. A 50, 135 (2008).

3. S. A. Vshivkov and E. V. Rusinova, Zh. Prikl. Khim. (S.-Peterburg) 84, 1739 (2011).
4. S. A. Vshivkov, A. Yu. Zubarev, and A. P. Safronov, SelfOrganization, Phase Transitions and Properties of Anisotropic Media in Magnetic and Mechanical Fields (AMB, Yekaterinburg, 2011) [in Russian].

5. S. A. Vshivkov and E. V. Rusinova, Polymer Science, Ser. A 50, 725 (2008).

6. S. A. Vshivkov, A. G. Galyas, L. I. Kutsenko, I. S. Tyukova, T. V. Terziyan, and A. V. Shepetun, Polymer Science, Ser. A 53, 1 (2011).

7. S. A. Vshivkov and A. G. Galyas, Polymer Science, Ser. A 53, 1032 (2011).

8. V. G. Kulichikhin and L. K. Golova, Khim. Drev., No. 3, 9 (1985).

9. J. T. Yang, J. Am. Chem. Soc. 80, 1783 (1958).

10. J. T. Yang, J. Am. Chem. Soc. 81, 3902 (1959).

11. J. Hermans, Jr., J. Colloid Sci. 17, 638 (1962).

12. V. G. Kulichikhin, V. A. Platonov, E. G. Kogan, L. P. Mil'kova, I. N. Andreeva, N. V. Luasheva, A. V. Volokhina, and S. P. Papkov, Vysokomol. Soedin., Ser. A 20, 2224 (1978).

13. S. P. Papkov, V. G. Kulichihin, V. D. Kalmykova, and A. Ya. Malkin, J. Polym. Sci., Part B: Polym. Phys. 12, 1753 (1974).

14. B. V. Ioffe, Refractometric Techniques in Chemistry (Khimiya, Leningrad, 1974) [in Russian].

15. S. A. Vshivkov, E. V. Rusinova, N. V. Kudrevatykh, A. G. Galyas, M. S. Alekseeva, and D. K. Kuznetsov, Polymer Science, Ser. A 48, 1115 (2006).

16. W. Heller, H. L. Bhathagar, and M. Nakagaki, J. Chem. Phys. 36, 1163 (1962).

17. W. Heller and W. L. Pangonis, J. Chem. Phys. 26, 498 (1957).

18. V. I. Klenin, S. Yu. Shchegolev, and V. I. Lavrushin, Characteristic Functions of Light Scattering in Disperse Systems (Saratovsk. Gos. Univ., Saratov, 1977) [in Russian].

19. D. W. Van Krevelen, Properties of Polymers: Their Estimation and Correlation with Chemical Structure (Elsevier, Amsterdam, 1972; Khimiya, Moscow, 1976).

20. Yu. S. Lipatov and A. E. Nesterov, Handbook on Polymer Chemistry (Naukova Dumka, Kiev, 1971) [in Russian].

21. E. V. Kitaev and N. F. Grevtsev, General Electric Engineering (Sovetskaya Nauka, Moscow, 1945) [in Russian].

22. N. I. Koshkin and M. G. Shirkevich, Handbook on Elementary Physics (Nauka, Moscow, 1988) [in Russian].

23. W. G. Miller, C. C. Wu, E. L. Wee, G. L. Santee, J. H. Rai, and K. D. Goebel, Pure Appl. Chem. 38, 37 (1974).

24. S. A. Vshivkov, E. V. Rusinova, and A. G. Galyas, Polymer Science, Ser. A 54, 827 (2012). 